\title{
PESSOAS COM ÚLCERAS VENOSAS: estudo do modo psicossocial do Modelo Adaptativo de Roy
}

I sabelle Katherinne Fernandes COSTA A , Walkíria G omes da N Ó BRE GA A, I sabel Karolyne Fernandes COSTAc, Gilson de Vasconcelos TORRESd, A na Luiza Brandão de Carvalho LIRAe,

Francis Solange Vieira T OU RI N HO ${ }^{f}$, Bertha Cruz E N DERS ${ }^{g}$

\section{RESUMO}

E studo transversal, descritivo, quantitativo, realizado com 50 pessoas com úlcera venosa (UV) em um Hospital U niversitário, que objetivou verificar o nível de adaptação psicossocial do M odelo de Roy das pessoas com UV. A pesquisa foi aprovada pelo Comitê de É tica da instituição. Os dados foram coletados com formulário estruturado e após a organização dos dados das alterações ocorridas na vida das pessoas com U V classificamos segundo o modo psicossocial de Roy. Verificamos que no modo autoconceito, 36\% sentiam-se insatisfeitos com aparência física, 18\% apresentavam sentimentos negativos; no modo desempenho de papéis: alter ação no papel laboral $(52,0 \%)$, atividades domésticas (34,0\%), conjugal $(6,0 \%)$, restrições de lazer, dor, social, escolar e locomoção (82,0\%); no modo de interdependência: apoio no tratamento (82,0\%), discriminação (58,0\%). A identificação do modo psicossocial direciona as ações de enfer magem abrangendo a pessoa que recebe o cuidado consider ando-a no todo em suas relações com ambiente promovendo melhor nível de adaptação.

D escritores: Ú Icera varicosa. T eoria de enfermagem. M odelos de enfer magem.

\section{RESUMEN}

E studio transversal, descriptivo, cuantitativo realizado con 50 personas con úlcera venosa (U V) en el H ospital U niversitario que objetivó conocer nivel de adaptación psicosocial del M odelo de R oy de las personas con U V. E studio aprobado por el Comité de É tica. L os datos fueron recogidos mediante formulario y después de organizar los datos de los cambios en la vida de las personas con UV, fueron clasificados de acuerdo con los aspectos psicosociales de R oy. Verificamos en el modo de autoconcepto: $36 \%$ insatisfacción con apariencia física, 18\% sentimientos negativos, desempeño de papel: cambio en el papel de trabajo $(52,0 \%)$, tareas domésticas $(34,0 \%)$, estado civil $(6,0 \%)$, restricción de ocio, dolor, sociales, educativos y transporte $(82,0 \%)$; modo de interdependencia: a poyo en el tratamiento $(82,0 \%)$, discriminación $(58,0 \%)$. L a identificación de aspectos psicosociales dirige las acciones de enfermería cubriendo estas personas que reciben atención en la totalidad de sus relaciones con el medio ambiente promoviendo mayor nivel de adaptación.

D escriptores: Ú Icera varicosa. T eoría de enfermería. M odelos de enfermería.

T ítulo: P ersonas con úlceras venosas: estudio de los aspectos psicosociales del M odelo de A daptación de R oy.

a M estre em Enfer magem, D outoranda pelo Programa de Pós-G raduação em E nfermagem da U niver sidade Federal do Rio G rande do N orte (UFRN), Bolsista da Coordenação de A perfeiçoamento de Pessoal de N ível Superior (CAPES), N atal, Rio G rande do N orte, Brasil.

b M estre em Enfermagem, Enfermeira do Serviço de A tendimento M óvel de U rgência (SAM U) 'M etropolitano, N atal, Rio G rande do N orte, Brasil.

'Enfermeira, M estranda do Programa de Pós-Graduação em Enfermagem da UFRN, Enfermeira do SAM U M etropolitano, Natal, Rio Grande do N orte, Brasil.

d D outor em Enfermagem, Professor T itular do Departamento de Enfermagem da UFRN, Bolsista de Produtividade em Pesquisa PQ2 do Conselho N acional de Desenvolvimento Científico e Tecnológico (CN Pq) (2009/2012), N atal, Rio G rande do N orte, Brasil.

e D outora em Enfermagem, Professora A djunta I do D epartamento de Enfermagem e do Programa de Pós-G raduação em Enfermagem da U F R N, N atal, Rio G rande do N orte, Brasil.

† D outora em Saúde da Criança e do Adolescente, Professora do Departamento de Enfermagem da UFRN, Natal, Rio Grande do Norte, Brasil.

9 Enfermeira, Professora T itular do D epartamento de Enfermagem da UFRN, Bolsista de Produtividade em Pesquisa do CN Pq - Nível 2 , Natal, Rio Grande do N orte, Brasil. 


\section{ABST RACT}

A transversal descriptive quantitative study, conducted with 50 people with Venous U Icer (VU ) at an U niversity H ospital, that aimed to ascertain the level of psychosocial adaptation of the R oy M odel of people with VU. The study was approved by the E thics Committee. The data were collected with a structured form. After organizing the data, composed of changes in the lives of people with VU, we classified it according to R oy's psychosocial aspects. We found that in the self-concept mode, $36 \%$ felt dissatisfied with physical appearance, and $18 \%$ had negative feelings; in the role function mode: change in the working role $(52.0 \%)$, housew ork $(34.0 \%)$, marital $(6.0 \%)$, leisure, pain, social, educational and transportation restrictions $(82.0 \%)$; interdependence mode: support in treatment (82.0\%), discrimination (58.0\%). The identification of the psychosocial aspects directs nursing actions to consider the whole of the person receiving care in its relations with the environment, thus promoting a better level of adaptation.

Descriptors: Varicose ulcer. N ursing theory. M odels, nursing.

T itle: P eople with venous ulcers: a study of the psychosocial aspects of the R oy A daptation M odel.

\section{INT RODUÇÃO}

Os avanços tecnológicos e científicos na área da saúde, bem como o processo de transição demográfico-epidemiológica possibilitaram, sem dúvida, 0 aumento da expectativa de vida da população brasileira nestes últimos anos. No entanto, em pleno século XXI, ainda são inúmeros e relevantes os problemas que continuam a afetar a saúde das pessoas no mundo e no Brasil. Essas repercussões do desenvolvimento científico e tecnológico nas condições de vida da população vêm preocupando os cientistas, não somente com a efetividade das inter venções terapêuticas, mas também com as meIhorias obtidas na Qualidade de Vida (QV) dos pacientes, principal mente dos que apresentam as doenças degenerativas e não degener ativas(1).

0 impacto na mortalidade, morbidade e altos custos, as doenças crônicas não-transmissíveis vêm se tornando prioridade na maioria dos países. No Brasil, ainda hoje são pouco conhecidos a incidência dessa parcela de doenças e os fatores de risco associados, apesar das estatísticas oficiais de mortalidade, dos dados de vigilância epidemiológica de doenças crônicas e dos estudos que determinam a frequência de fatores de risco para essas doença ${ }^{(2)}$. Sendo assim, de extrema importância as informações sobre a incidência das doenças crônicas para o desenvolvimento de programas preventivos e a formulação de políticas públicas que reduzam o impacto dessas doenças no País(2).

A Insuficiência Venosa Crônica (IVC) é uma das enfermidades crônicas que afeta o homem, estando relacionada à presença de hipertensão venosa prolongada que se desenvolve quando a pressão venosa está aumentada e o retorno do sangue é diminuído através de vários mecanismos, podendo ser resultado da incompetência valvular das veias superficiais e profundas, da obstrução venosa, ou uma combinação destes. Esses fatores são agravados pela disfunção no músculo da panturriIha ${ }^{(3)}$. A pesar de a mortalidade ser praticamente inexistente, apresenta elevada morbidade e é caracterizada principal mente pela ocorrência de Ú Icera Venosa (UV) nos membros inferiores, quando em estágio avançado(4).

A UV apresenta-se como complicação mais séria da IVC, é caracterizada pela perda irregular do tegumento de forma superficial, podendo se tornar profunda, bordas definidas, comumente com exudato amarelado, iniciando de forma espontânea ou traumática. As úlceras podem ser únicas ou múltiplas, de tamanhos e localizações variáveis, em geral ocorre no terço médio distal da perna, com maior incidência nas proeminências ósseas, especialmente nos maléolos mediais, podendo envolver toda a circunferência da perna se não tratada(5).

Esse tipo de lesão de perna apresenta-se com alta prevalência, caráter recidivante, o que provoca sofrimento tanto ao paciente como a sua família. Além de gerar dependência dos serviços de saúde, constituindo um importante problema de saúde pública, e assumir uma importante magnitude no que se refere à repercussão social e econômica em ter mos de dias de trabalho perdidos, acarreta uma diminuição da qualidade de vida(1,4).

0 impacto da alteração do estilo de vida da população acometida pelas úlceras atinge diretamente a qualidade de vida, uma vez que esta é marcada pela subjetividade, e envolve todos os componentes essenciais da condição humana, quer 
seja físico, psicológico, social, cultural ou espiritual(1).

Viver com a condição de ter uma ferida acarreta uma série de mudanças na vida das pessoas e, consequentemente, de seus familiares ${ }^{(6)}$. $\mathrm{N}$ esta situação aparecem dificuldades que muitas vezes nem o paciente e nem a família estão preparados para ajudar e compreender todos os aspectos que envolvem o problema.

Surge, então, a necessidade de adaptação dos pacientes com UV sob a sua nova condição de vida que compromete seu bem estar mental, físico e social. T udo isso gera a necessidade de obtenção de novas habilidades incluindo revisão de valores, conhecimento científico e prático sobre a enfermidade, adaptação ao tratamento, além do enfrentamento da sociedade.

Comportamentos como os apresentados por esses pacientes com relação à sua enfermidade são abordados pela teoria de Roy em quatro modos de adaptação: o modo fisiológico; o modo de autoconceito; o modo de desempenho de papéis e o modo de interdependência. Este trabalho se propôs a estudar os três últimos modos que, juntos, constituem o modo psicossocial (7).

0 modo do autoconceito evidencia aspectos psicológicos e espirituais do ser humano. Esse modo refere-se à fusão de crenças e sentimentos de um indivíduo em determinada circunstância. 0 modo de desempenho de papéis explora padrões de interação pessoal, destacando os fatores relacionados aos papéis que o sujeito cumpre no meio social em que vive, bem como seu desempenho. 0 modo de interdependência destaca as interações de dar e receber afeto, respeito e valor. É considerado um modo social por que suas necessidades são atendidas por meio da interação social ${ }^{(7-9)}$.

Considerando que o ser humano é um sistema biopsicossocial com a capacidade de ajustarse ao ambiente bem como modificá-lo, buscamos neste estudo verificar o nível de adaptação psicossocial dos portadores de UV, a fim de se compreender de que forma essas pessoas estão enfrentando a condição de ter uma doença crônica.

Por acreditarmos que a UV, surge em decorrência da falta de adaptação do cliente à doença e ao tratamento, neste estudo trabal haremos com 0 modelo conceitual de enfermagem proposto por Roy e Andrews, conhecido como M odelo de Adaptação, com o objetivo de verificar o nível de adaptação psicossocial destas pessoas ${ }^{(7)}$.

\section{MÉTODOS}

Trata-se de um estudo transversal, descritivo de abordagem quantitativa realizado em um Hospital U niversitário localizado no município de $\mathrm{N}$ atal no Rio Grande do Norte (RN), o qual pertence ao Complexo de Saúde da U niversidade Federal do Rio Grande do N orte (UFRN), especificamente no ambulatório da clínica cirúrgica, com atendimento em angiologia e cirurgia vascular.

A amostra foi composta por 50 pacientes portadores de UV, selecionados a partir de uma amostra de acessibilidade, com base nos critérios de inclusão a seguir: ser portador de UV; ter mais de 18 anos; e ser atendido no ambulatório de clínica cirúrgica do referido hospital no período da coleta de dados. Critérios de exclusão: paciente que não concluiu a coleta de dados, por motivo de ausência do setor no momento da coleta e o paciente que não apresentou condições cognitivas de responder os instrumentos.

A pesquisa foi submetida à apreciação do Comitê de Ética e Pesquisa do referido hospital (protocolo nํ 279/09), respeitando a normatização da Resolução 196/ 96 do Conselho N acional de Saúde, no que se refere aos aspectos éticos observados quando da realização da pesquisa envolvendo seres humanos ${ }^{(10)}$.

0 processo de coleta de dados ocorreu entre os meses de maio a julho de 2009. Os dados foram coletados através da aplicação de um formulário estruturado contendo dados sócio-demográficos, de saúde, clínicas, assistenciais do paciente, e referentes ao modo de adaptação psicossocial de Roy.

Os pacientes foram abordados durante a troca de curativos e convidados a participarem da pesquisa, após uma explanação sobre o objetivo do estudo e obtenção da assinatura do T ermo de Consentimento Livre e Esclarecido (TCLE).

Os dados coletados foram organizados em um banco de dados eletrônico do aplicativo M icrosoft Excel e analisados no programa Statistical Package for the Social Sciences (SPSS) ver são 15.0, sendo codificados, tabulados e apresentados na forma de tabelas e quadros com suas respectivas distribuições percentuais. Para tratamento dos dados utilizou-se estatística descritiva.

A pós a organização dos dados referentes às alterações ocorridas na vida referidas pelas pessoas com UV neste estudo, os classificamos segundo os modos adaptativos do M odelo de A daptação de R oy. 


\section{RESULTADOS E DISCUSSÕES}

$\mathrm{Na}$ T abela 1 apresentamos a distribuição da caracterização sócio-demográfica dos 50 portadores de U V, com predomínio de pacientes do sexo feminino $(76,0 \%)$ e faixa etária menor do que 59 anos (74,0\%). No tocante ao grau de escolaridade, $68,0 \%$ tinham baixa escolaridade, dos quais $76,0 \%$ eram mulheres.

Em relação ao estado civil, $62,0 \%$ dos pesquisados eram casados ou mantinham união estável, e $22,0 \%$ dos pacientes eram solteiros. Sobressaiu a religião católica (68,0\%). E m relação ao número de filhos, $38,0 \%$ dos pesquisados tinham mais de três filhos, desses $42,0 \%$ do sexo feminino.

$\mathrm{N}$ o que diz respeito à vida labor al, $70,0 \%$ não possuíam vínculo empregatício, 72,0\% tinham uma renda de até dois salários mínimos e 32,0\% mudaram sua ocupação em detrimento ao surgimento da úlcera venosa.

Estudos nacionais e inter nacionais revelam que há uma predominância do sexo feminino nos pacientes com UV, representando uma relação de 3:1 entre os sexos ${ }^{(11,12)}$.

T abela 1 - Caracterização sócio-demográfica das 50 pessoas com úlcera venosa (U V) segundo o tempo de lesão atual. Natal, R N , 2009.

\begin{tabular}{|c|c|c|c|c|c|c|}
\hline \multirow{3}{*}{ Caracterização sócio-demográfica } & \multicolumn{4}{|c|}{ Sexo } & & \\
\hline & \multicolumn{2}{|c|}{ M asculino } & \multicolumn{2}{|c|}{ Feminino } & \multicolumn{2}{|c|}{ T otal } \\
\hline & $\bar{n}$ & $\%$ & $\mathbf{n}$ & $\%$ & $\mathrm{n}$ & $\%$ \\
\hline \multicolumn{7}{|l|}{ Faixa etária } \\
\hline A té 59 anos & 11 & 22,0 & 26 & 52,0 & 37 & 74,0 \\
\hline A partir de 60 anos & 1 & 2,0 & 12 & 24,0 & 13 & 26,0 \\
\hline \multicolumn{7}{|l|}{ E stado civil } \\
\hline Casado & 9 & 18,0 & 22 & 44,0 & 31 & 62,0 \\
\hline Solteiro & 3 & 6,0 & 8 & 16,0 & 11 & 22,0 \\
\hline Divorciado/Separado & - & - & 3 & 6,0 & 3 & 6,0 \\
\hline Viúvo & - & - & 5 & 10,0 & 5 & 10,0 \\
\hline \multicolumn{7}{|l|}{ E scolaridade } \\
\hline A té ensino fundamental & 5 & 10,0 & 29 & 58,0 & 34 & 68,0 \\
\hline Ensino médio & 7 & 14,0 & 8 & 16,0 & 15 & 30,0 \\
\hline Ensino superior & - & - & 1 & 2,0 & 1 & 2,0 \\
\hline \multicolumn{7}{|l|}{ Religião } \\
\hline Católico & 8 & 16,0 & 25 & 50,0 & 33 & 66,0 \\
\hline Protestante & 4 & 8,0 & 13 & 26,0 & 17 & 34,0 \\
\hline \multicolumn{7}{|l|}{ №filhos } \\
\hline A té 03 filhos & 9 & 18,0 & 22 & 44,0 & 31 & 62,0 \\
\hline A cima de 03 filhos & 3 & 6,0 & 16 & 32,0 & 19 & 38,0 \\
\hline \multicolumn{7}{|l|}{ Vínculo empregatício } \\
\hline Sim & 8 & 16,0 & 7 & 14,0 & 15 & 30,0 \\
\hline Não & 4 & 8,0 & 31 & 62,0 & 35 & 70,0 \\
\hline \multicolumn{7}{|l|}{ Renda } \\
\hline A té 02 salários & 8 & 18,0 & 28 & 56,0 & 36 & 72,0 \\
\hline M ais de 02 salários & 4 & 6,0 & 10 & 20,0 & 14 & 28,0 \\
\hline M udança de ocupação por causa da UV & 5 & 10,0 & 11 & 22,0 & 16 & 32,0 \\
\hline$M$ ais de 03 refeições & 5 & 10,0 & 16 & 32,0 & 21 & 42,0 \\
\hline
\end{tabular}

A utores já sinalizam um crescente número de adultos jovens com ulceração venosa em perna de condição crônica, apesar da prevalência desta patologia ser maior na população idosa ${ }^{(13)}$.

Estudo realizado em 2003 aponta que aproximadamente $22,0 \%$ das pessoas que desenvolvem úlceras venosas, foram acometidas por volta dos
40 anos de idade e $13,0 \%$ antes dos 30 anos de idade, o que representa um substancial efeito sobre a produtividade do trabalho(14).

De acordo com alguns autores, há evidências de que a baixa renda influencia de maneira negativa o comportamento saudável no ambiente domiciliar, o acesso aos serviços de saúde, os cui- 
dados com a saúde e o acesso aos recursos materiais $^{(1,14)}$.

A diminuição da QV dos pacientes, entre outros fatores, está fortemente associada à perda de dias de trabalho, que gera aumento de atestados médicos e até mesmo 0 desemprego ${ }^{(13)}$.

A lém disso, os indivíduos acometidos pela UV necessitam se afastar do trabalho inúmeras vezes e com frequencia, são aposentados precocemente, pelo fato destas lesões exigirem terapêuticas prolongadas, causando um importante ônus aos sistemas de saúde e previdenciário; além dos incalculáveis custos humanos associados à necessidade de ajustar as atividades no trabalho, perda dos dias de trabal ho ou do emprego, aumento da demanda por recursos de saúde e diminuição do prazer nas atividades da vida cotidiana(15,16).

A partir do Quadro 1 percebemos diversos fatores sócio-demográficos, clínicos e assistenciais que sofrem influência da úlcera venosa e refletem na vida dos pacientes.

Corroborando com o estudo, alguns autores referem que a cronicidade da U V atinge os indivíduos em sua fase mais produtiva da vida, acarretando dor, perda de mobilidade e afastamento de atividades, 0 que interfere diretamente na QV ${ }^{(16)}$.

\begin{tabular}{|c|c|}
\hline Categorias & D escrição \\
\hline $\begin{array}{l}\text { Lazer/ dor/ restrição social/ } \\
\text { escolar/ locomoçãa }\end{array}$ & $\begin{array}{l}\text { Não frequenta mais a igreja, supermercado, feiras, clubes, grupo de igre- } \\
\text { ja, procissões, sair com amigos, diminuiu o ciclo de amizades, isolamento } \\
\text { social, não pode sair só, visitar parentes no hospital, deixou de sair de } \\
\text { casa devido a dores, dificuldade de se locomover, deixou de ir ao shopping, } \\
\text { deixou de estudar, morava num sítio, onde se sentia mais feliz, e teve que } \\
\text { vir para a cidade. }\end{array}$ \\
\hline Sentimentos negativos & $\begin{array}{l}\text { Depressão, expectativas negativas, preocupação, pensativa, tristeza, per- } \\
\text { da de ânimo, sentimento de inutilidade, de prisão, ag ressividade, impaci- } \\
\text { ência, medo de amputação. }\end{array}$ \\
\hline $\begin{array}{l}\text { Restrição atividade } \\
\text { doméstica }\end{array}$ & $\begin{array}{l}\mathrm{N} \text { ão pode real izar os afazeres domésticos, devido a dores, tem que se sub- } \\
\text { meter à ajuda de al guém para real izar as tar efas. }\end{array}$ \\
\hline Restrição conjugal & $\begin{array}{l}\text { Deixou de ter relações sexuais, terminou um relacionamento (devido à } \\
\text { úlcera - vergonha), separação conjugal. }\end{array}$ \\
\hline $\begin{array}{l}\text { Restrição financeira/ laboral/ } \\
\text { progressão profissional }\end{array}$ & $\begin{array}{l}\text { Diminuição da renda familiar, aumento de gastos com remédios, não pode } \\
\text { trabal har, atestado médico no trabal ho, diminuiu o rendimento no traba- } \\
\text { lho, dificuldade de trabal har, teve que ficar na perícia, dificultou a oportu- } \\
\text { nidade de trabal ho, não trabal ha mais onde gosta, não pode real izar con- } \\
\text { cursos públicos que exijam exames físicos. }\end{array}$ \\
\hline A parência física/ discriminação & $\begin{array}{l}\text { Vergonha da lesão, vestimentas sempre cobrindo os membros inferiores, } \\
\text { distúrbio da auto-imagem, vergonha, exsudato, constrangimento, curio- } \\
\text { sidade al heia sobre a lesão, incomodo com o odor da lesão, motivo de } \\
\text { zombaria, aspecto da lesão. }\end{array}$ \\
\hline
\end{tabular}

Q uadro 1 - Descrição das categorias das alterações na vida das 50 pessoas com úlcera venosa (UV). N atal, R N, 2009.

As discussões das vivências desses pacientes em relação à úlcera venosa crônica foram aprofundadas com os modos de adaptação psicossocial autoconceito, desempenho de papéis e interdependência de R oy que serão discutidos a seguir.

A T abela 2 seguinte apresenta as categorias que se enquadram no modo autoconceito, em que $36 \%$ dos entrevistados sentiam-se insatis- feito com sua aparência física ou se sentiam discriminados, e $18 \%$ apresentavam sentimentos negativos, devido a falta de adaptação com a doença e com o desenvol vimento de uma úlcera venosa crônica.

0 autoconceito é um dos três modos psíquico social que enfoca especificamente os aspectos psicológicos e espirituais da pessoa ${ }^{(7)}$. 
T abela 2 - M odo do autoconceito de Roy segundo alterações na vida das 50 pessoas com Ú Icera Venosa. Natal, R N , 2009.

\begin{tabular}{lrrrr}
\hline \multirow{2}{*}{ M odo do Autoconceito } & \multicolumn{2}{c}{ Sim } & \multicolumn{2}{c}{ Não } \\
\cline { 2 - 5 } & $\mathbf{n}$ & $\%$ & $\mathbf{n}$ & $\%$ \\
\hline A parência física/ & & & & \\
Discriminação & 18 & 36,0 & 32 & 64,0 \\
Sentimentos negativos & 9 & 18,0 & 41 & 82,0
\end{tabular}

Observou-se que a insatisfação dessas pessoas, frente às sensações corporais, aponta para um baixo sentido de sua autovalorização. E sse sentimento pode diminuir a habilidade dessas pessoas para enfrentamentos das situações. Por outro lado, a imagem corporal significa a percepção da pessoa sobre o seu corpo. N essa categoria, detectou-se comportamento inefetivo em $36,0 \%$ dos pesquisados.

$\mathrm{Na} U \mathrm{~V}$, a lesão nos membros inferiores, pode levar a pessoa a sentir que sofreu perda de partes ou função do corpo. Esse fato pode conduzi-la, também, a questionar seu valor, alterando, dessa forma, seu autoconceito físico. Se uma pessoa tem bai$x a$ auto-estima, ela tende a perceber 0 meio ambiente como negativo e ameaçador ${ }^{(17)}$.

A auto-estima é o aspecto penetrante do componente eu pessoal, que se relaciona ao valor que a pessoa tem de si, e a redução da auto-estima é vista como um sentimento negativo de desânimo, desvalorização e isolamento 0 que dificulta a capacidade de uma pessoa para se adaptar ao meio ambiente ${ }^{(18)}$. Comumente, os valores contribuintes para a baixa auto-estima são as limitações físicas e as mudanças corporais ocorridas, nesse caso, relacionada a presença da lesão(19).

$\mathrm{Na}$ T abela 3, temos as categorias que apresentaram alterações no modo desempenho de papéis, que focaliza os papéis ocupados pela pessoa na sociedade, buscando a integ ridade social da pessoa ${ }^{(7)}$.

Quanto aos papéis, estes podem ser classificados como primários, secundários e terciários. 0 papel primário é determinado pela idade, sexo e estado desenvolvimental ${ }^{(7)}$.

No presente estudo, observamos que os entrevistados, em relação ao seu papel primário, encontravam-se no estágio desenvolvimental correspondente a idade adulta (até 59 anos) e início da senilidade (a partir dos 60 anos).

0 papel secundário é aquele assumido pela pessoa para complementar os deveres associados com o estado desenvolvimental e o papel primário ${ }^{(7)}$. Quanto aos papéis secundários, os pesquisados apresentaram essencialmente os papéis de mãe, pai, marido, mulher e responsáveis pela renda familiar.

T abela 3 - M odo do desempenho de papéis de R oy segundo alterações na vida das 50 pessoas com Ú Icera Venosa. N atal, R N , 2009.

\begin{tabular}{lrrrr}
\hline M odo do desempenho & \multicolumn{2}{c}{ Sim } & \multicolumn{2}{c}{ Não } \\
\cline { 2 - 5 } de papéis & $\mathbf{n}$ & $\%$ & n & \% \\
\hline Lazer/dor/ restrição social/ & & & & \\
escolar/locomoção & 41 & 82,0 & 9 & 18,0 \\
$\begin{array}{l}\text { Restrição laboral/ financeira/ } \\
\text { progressão social }\end{array}$ & 26 & 52,0 & 24 & 48,0 \\
Restrição de atividade domés- & & & & \\
tica & 17 & 34,0 & 33 & 66,0 \\
Restrição conjugal & 3 & 6,0 & 47 & 94,0 \\
\hline
\end{tabular}

Observamos, contudo, alteração nos papéis laboral $(52,0 \%)$, atividades domésticas $(34,0 \%)$ e conjugal $(6,0 \%)$.

0 papel terciário é de livre escolha da pessoa, temporário e natural, podendo incluir atividades como clubes e passatempos ${ }^{(7)}$. Quanto aos papéis terciários, verificamos restrições relacionadas ao lazer, dor, restrição social, escolar e relacionada a locomoção (82,0\%).

Conviver com a insuficiência venosa crônica, e, principalmente com a úlcera venosa crônica é um processo de aprendizagem, 0 qual requer tempo. M uitas pessoas enfrentam melhor se forem ativas, especial mente quando se deter minam a fazer atividades, adaptando-se as suas limitações, promovendo uma melhor QV.

No que diz respeito ao modo de interdependência, temos aqueles pacientes que receberam ou não apoio no tratamento, o que os que sentem que sofreram algum tipo de discriminação, devido a presença da úlcera, sendo explanado na T abela 4.

0 modo de interdependência envolve interação com as outras pessoas, focalizando relacionamentos íntimos implicados em papéis ou na posição na sociedade ${ }^{(7)}$.

A enfermidade, de certa forma, é compartiIhada por toda família. Desta forma, quando os familiares estão presentes apoiando-os o processo de enfrentamento é compartilhado e possibilita um processo de adaptação(19,20).

A maneira de reagir frente à doença difere de pessoa para pessoa. Alguns buscam apoio na fé, 
outros nos amigos e familiares e outros, nos profissionais de saúde ${ }^{(19)}$.

T abela 4 - M odo da interdependência de Roy segundo alterações na vida das 50 pessoas com Úlcera Venosa. N atal, RN, 2009.

\begin{tabular}{lrrrr}
\hline $\begin{array}{l}\text { M odo da Inter- } \\
\text { dependência }\end{array}$ & \multicolumn{2}{c}{ Sim } & \multicolumn{2}{c}{ Não } \\
\cline { 2 - 5 } & $\mathbf{n}$ & $\%$ & $\mathbf{n}$ & $\%$ \\
\hline Recebe apoio no & & & & \\
tratamento & 41 & 82,0 & 9 & 18,0 \\
$\quad$ Família & 23 & 46,0 & 27 & 54,0 \\
A migos & 16 & 32,0 & 34 & 68,0 \\
$\quad$ Sociedade & 28 & 56,0 & 22 & 44,0 \\
D iscriminação & 29 & 58,0 & 21 & 42,0 \\
$\quad$ Família & 23 & 46,0 & 27 & 54,0 \\
A migos & 8 & 16,0 & 42 & 84,0 \\
Sociedade & 7 & 14,0 & 43 & 86,0 \\
\hline
\end{tabular}

No entanto, é importante destacar que nas famílias, os comportamentos relacionados à pessoa com UV, em sua maioria, não foram adaptativos, visto que a maioria não recebe apoio no tratamento e ainda sofrem discriminação principalmente da família. As relações de interdependência, em geral, sofreram al terações negativas, o que demonstra um enfrentamento insatisfatório do problema, necessitando, portanto de um apoio maior e orientações.

Diante deste resultado observamos que a falta da adaptação ao tratamento dentro dos modos de autoconceito, desempenho de papéis e de interdependência, que constituem o modo psicossocial, contribui para a cronicidade das lesões, o que acarreta numa QV deteriorada.

N este contexto, o sistema de cuidado de saúde pode ser um sistema de suporte, em que os profissionais atuantes, no tratamento dos pacientes com úlcera venosa, empreguem esforços contínuos para o reconhecimento das necessidades humanas afetadas e, a partir delas, contribuam para a adaptação deles a sua nova condição de vida( ${ }^{(7,19)}$.

\section{CONCLUSÕES}

As pessoas do estudo apresentam um perfil com predominância no sexo feminino, com a faixa etária de 18 a 59 anos, de religião católica, com o nível de escolaridade baixo, em sua maioria casado, com até três filhos. Verificamos que o nível de adaptação psicossocial dessas pessoas é baixo em decorrência da dificuldade na adesão ao tratamento, contribuindo para a cronicidade dessas lesões, deteriorando ainda mais a QV dessas pessoas.

Nesse sentido, o cuidado de enfermagem visualizado a partir da ótica da teoria da adaptação de Roy possibilita um trabalho de efetivo desempenho. A identificação do modo psicossocial direciona as ações de enfermagem de forma a abranger a pessoa que recebe o cuidado considerando-a no todo em suas relações com ambiente inter no e externo. As metas visam ao restabelecimento e/ ou à manutenção do equilíbrio sendo possível relacioná-las às inter venções necessárias.

N este modelo, é válido salientar que alguns modos adaptativos, por se fazerem presentes no âmbito interno da pessoa, necessitam de um trabal ho mais cuidadoso englobando a participação de outras pessoas, sobretudo de familiares. Isto demonstra que os modos adaptativos psicossocial requerem um trabalho aprofundado de conscientização, educação e sensibilização constante, de forma a permitir uma reestruturação e equilíbrio da pessoa que recebe o cuidado.

Tratando-se de doença crônica, torna-se imprescindível que a clientela conheça sua doença para saber o quê e quando esper ar em cada situação, colaborando, dessa for ma, com sua própria adaptação.

Para tanto, sugere-se a abordagem terapêutica interdisciplinar em saúde, formada por grupo de profissionais (enfermeiros, médicos, psicólogos, assistentes sociais, dentre outros) para assistência aos clientes com úlcera venosa, em seus diversos aspectos, buscando seu melhor nível de adaptação.

Ressalta-se o papel peculiar do enfermeiro como educador junto a esta clientela, com intuito de orientar os pacientes a procurar meios adaptativos, que os façam superar sua condição clínica, com vistas a uma recuperação efetiva e melhoria da sua QV.

\section{REFERÊNCIAS}

1 N unes J P, Vieira D, N óbrega W G, F arias TYA, T orres $G V$. Venous ulcers in patients treated at family health units in $\mathrm{N}$ atal, Brazil: prevalence and sociodemographic and health characterization. FIE P Bull. 2008;78(1):338-41.

2 F rade M AC, Cursi IB, Andrade F F, Soares SC, Ribeiro W S, Santos SV, et al. Úlcera de perna: um estudo de casos em Juiz de Fora-M G (Brasil) e região. An Bras Dermatol. 2005;80(1):41-6. 
3 M inistério da Saúde (BR), Secretaria de Políticas de Saúde, D epartamento de A tenção Básica. M anual de condutas para úlceras neurotróficas e traumáticas. Brasília (D F); 2002.

4 França LH G, T avares V. Insuficiência venosa crônica: uma atual ização. J Vasc Bras. 2003;2(4):318-28.

5 Silva JL A, Lopes M JM . E ducação em saúde a portadores de úlcera varicosa através de atividades de grupo. Rev G aúcha Enferm [ I nternet] . 2006 [ citado 2010 jul 06] ;27(2):240-50. Disponível em http:/ / www.seer. ufrgs.br/ index.php/ RevistaG auchadeE nfermagem/ article/ view/4602/ 2522.

6 L ucas LS, M artins JT, Robazzi M LCC. Qualidade de vida dos portadores de ferida em membros inferiores: úlcera de perna. Ciênc E nferm. 2008;14(1):43-52.

7 Roy C, Andrews HA. T he Roy A daptation M odel. $2^{\text {nd }}$ ed. N orwalk: A ppleton e Lange; 1999.

8 Carvalho ALB, Guedes M VC, Lopes M VO. A daptação psicossocial do adolescente pós-transplante renal segundo a teoria de Roy. Invest E duc Enferm. 2005;23(1):68-77.

9 Rodrigues D P, Pagliuca L M F, Silva RM . M odelo de Roy na enfermagem obstétrica: análise sob a óptica de M el eis. Rev G aúcha E nferm [ I nter net] . 2004 [ citado 2010 jul 06] ;25(2):165-75. D isponível em: http:/ / www.seer.ufrgs.br/index.php/ RevistaG auchade E nfermagem/ article/ view/ 4503/ 2440.

10 M inistério da Saúde (BR ), Conselho N acional de Saúde. Resolução 196, de 10 de outubro de 1996: diretrizes e normas regulamentadoras de pesquisa envolvendo seres humanos. Brasília (D F ); 1996.

11 Castillo PD, Sagues CR, U rrea RC, Bardisa M J, L opez SA. Colgajo sural en úlceras venosas crónicas de piernas. Rev Chil Cir. 2004;56(5):475-80.
12 Baptista CM C, Castilho V. Levantamento do custo do procedimento com bota de U nna em pacientes com úlcera venosa. Rev Latino-Am Enfermagem. 2006;14(6):944-9.

13 Nóbrega W G, M elo GSM, Costa IKF, D antas DV, $M$ acedo $E A B$, Torres $G V$, et al. M udanças na qualidade de vida de pacientes com úlcera venosa atendidos no ambulatório de um hospital universitário. Rev Enferm UF PE. 2011;5(2):220-7.

14 Ú Icer as venosas e arteriais. In: H ess CT. T ratamento de feridas e úlceras. 4aํ ed. São Paulo: Reichmann \& Affonso; 2002. p. 109-39.

15 Bergonese F N, Rivitti EA. Avaliação da circulação arterial pela medida do índice tornozelo/ braço em doentes de úlcera venosa crônica. An Bras D ermatol. 2006;81(2):131-5.

16 Farias SN P, Zeitoune RCG. A interferência da globalização na qual idade de vida no trabal ho: a percepção dos trabalhadores de enfermagem. Esc Anna N ery. 2004;8(3):386-92.

17 I vo M L, Carvalho EC. Assistência de enfermagem a portadores de anemia falciforme, à luz do referencial de Roy. Rev Latino-Am Enfermagem. 2003;11 (2):192-8.

18 Driver M J. Theory of self-concept. In: Roy C. Introduction to nursing: an adaptation model. Englewood Cliffs: Prentice-H all; 1984. p. 255-82.

19 Lira A L BC, G uedes CM V, L opes VOM . A daptação psicossocial do adolescente pós transplante renal segundo a teoria de Roy. Invest E duc Enferm. 2005; 23(1):68-77.

20 Barbosa JC, A guillar OM , Boemer M R. 0 significado de conviver com a insuficiência renal crônica. Rev Bras Enferm. 1999;52(2):293-302.

\section{Endereço da autora / Dirección del autor / Author's address:}

I sabelle Katherinne Fernandes Costa

Rua do M otor, 39, Praia do M eio

59010-090, N atal, R N

E-mail: isabellekfc@yahoo.com.br
Recebido em: 14/ 10/ 2010

A provado em: 30/ 06/ 2011 